\title{
Impact of Heterogeneous Deployment on Source Initiated Reactive Approach
}

\author{
Nonita Sharma *, Ajay K Sharma \\ Department of Computer Science \\ National Institute of Technology \\ New Delhi, India
}

\author{
Kumar Shashvat \\ Department of Computer Science \\ Chandigarh Group of Colleges \\ Mohali, India
}

\begin{abstract}
Selection of an optimal number of high energy level nodes and the most appropriate heterogeneity level is a prerequisite in the heterogeneous deployment of wireless sensor network, and it serves several purposes like enhanced network lifetime, finest energy consumption, and optimal sensing coverage. The paper presents the mathematical modeling of cost, energy and sensing range analysis of 2-level, 3-level, and n-level heterogeneous wireless sensor network. An experimental investigation has been carried out to investigate the effect of heterogeneity on a proposed Energy Efficient Source Initiated Reactive Algorithm. Studies on these aspects have been done to find the limitations of the algorithm for homogeneous networks and to find how it enriches sensing range and network lifetime. Based on the simulated experimental and numerical results, a mathematical model is presented to calculate the optimal number of high-level nodes which can simultaneously enhance network lifetime and achieve optimal sensing coverage. The results are compared with the homogeneous network to prove the effectiveness of the stated approach and proposed a model.
\end{abstract}

Keywords-Wireless sensor networks (WSNs); Cost Analysis Model; Energy Analysis Model; Sensing Range Model; Optimality

\section{INTRODUCTION}

Wireless Sensor Networks (WSNs) might be the fastest growing technology in our industry today. One of the ramifications of that growth is a dramatic increase in the number of applications exploring this technology and a proportionate surge in the number and types of research opportunities that could be evaluated in the future. The three primary classifications of applications in WSN are: sensor nodes forwarding data ceaselessly to the base station; base station commanding sensors to do something, and early warning systems.

Most of the classical approaches assume that the network deploys similar types of nodes regarding capabilities. However, there is a growing realization that the effect of heterogeneity needs to be explored and evaluated. Heterogeneity refers to a network that deploys nodes with different initial energy nodes. The use of heterogeneity has gained popularity because of their positive impact on network lifetime operation and enhanced energy efficiency. While the effects of heterogeneity can be evaluated after the deployment, pre-computing the optimal number of high-level nodes can make the network operations much efficient. Carrying the motivation of calculating the optimal number of high-end nodes one step further, different levels of heterogeneity are explored in heterogeneous networks. In this paper, three analysis models viz. Cost analysis, energy analysis, and sensing range analysis models for 2-level, 3-level, and n-level heterogeneous network are framed.

Also, if the number of high-end nodes increases in the network, inevitably, it will have a significant impact on the duration of network operation and network life time. But at the same time, increasing the number of high-end nodes in the network will have a direct impact on the overall cost of the network. And almost all applications work under a prescribed cost constraint. So, if there is a need to keep the cost factor same, then increasing the number of high-end nodes will lead to the decrease in the density of low-end nodes that will further impact the sensing coverage area and sensing coverage degree of the network. Sensing coverage area refers to the area that is being monitored by at least one functioning sensor in the network. Sensing degree refers to the number of total functioning nodes controlling the area. If the number of alive nodes that are monitoring the area is more, then more precise values can be generated. Hence, keeping the cost constraint in mind, a heterogeneous network needs to be explored for an optimal number of high-level nodes which can optimize both network lifetime and sensing coverage. Therefore, a mathematical model for optimality is presented to decide the optimal number of high-end nodes.

Further, selection of a particular routing algorithm depends on the characteristics of an application, which can be broadly be classified as event driven, continuous monitoring and query driven. Proactive routing strategy is applicable for continuous monitoring systems while reactive algorithms are the most suitable choices for event driven and query driven applications. In reactive algorithms, a path from sensor nodes sensing the events to the base station is formed only when events are occurring. Rest of the network operation, sensor nodes stay in sleep mode. Sleep mode helps in avoiding the energy consumption on maintaining the paths even during inactivity period. Hence, the proposed three models and mathematical analysis of optimality are simulated on a proposed reactive approach.

In this manuscript, an energy-efficient source initiated reactive (EE-SIR) approach is suggested that selects the parent node by several factors like residual energy, neighboring nodes, distance to the base station, stability values. Afterward, analysis of cost, energy and sensing range for different levels of heterogeneity on proposed EE-SIR routing strategy is presented as it has direct applications in real-life applications. The analysis is done for a direct communication model where 
every sensor is communicating directly with the sink. The case is presented for uniform distribution of nodes where high-end nodes are evenly distributed in the sensor area. An optimal number of heterogeneous nodes having a direct impact on network lifetime and sensing range is desired. Experiments have been conducted to create a mathematical model to calculate the optimal number of high-level nodes which can enhance network lifetime and achieve an optimal sensing range. Results demonstrate that an optimal number of high-end nodes can significantly improve the network operation, under the prescribed cost constraint. Besides, different heterogeneity levels and temporal correlation show different patterns over time, which will have a substantial effect on network performance and deployment decisions.

\section{RELATED WORK}

In our research work, authors work on finding the impact of adding different energy levels to a reactive routing protocol that can help to enhance the energy efficiency and sensing coverage under prescribed cost constraint.

As the number of nodes increases, homogeneous WSN is known to exhibit poor performance and reduced scalability. The throughput of a node is $\Theta(1 / \sqrt{n \log n)}$ where $\mathrm{n}$ is the number of nodes in the network [1]. Also, as paths between nodes turn out to be longer, the likelihood of packets being lost gets to be higher. Likewise, as the number of nodes in system advances, the packet delivery rate drops significantly. Experimentation results [2] and test beds [3] have shown a fading performance with increasing number of nodes.

Over the years, the impact of energy heterogeneity on the performance of a WSN has been a topical area of research. As per the definition given in [4], a heterogeneous sensor network is defined as a network of nodes with different functionalities and capabilities. For example, Mhatre et al. propose using two types of nodes; type 0 nodes acting as pure sensor nodes, and type 1 nodes that serve as the cluster head nodes [5]. Adding high capability nodes in the network has been considered to be an efficient and effective way to enhance reliability and network lifetime [6]. Along with, Mhatre et. al presented a survey showing that the heterogeneous WSN is more suitable for real-life applications as compared to their homogeneous counterpart [7]. Inculcating heterogeneity in the network enhanced response time and network lifetime. Heterogeneous HEED [8] showed a significant improvement in the lifetime just by introducing heterogeneity of various levels. Authors recommended nodes with different energy to prolong lifetime and reliability. Most of the time, the achievable lifetime could be optimized using multiple power levels. The paper concluded that the routing strategy should be such that the energy reserves should be the focus to balance the energy consumption; rather than minimizing the absolute consumed power to maximize the lifetime. However, in the current context of issues related to this area, more work can be done to improve energy efficiency.

More to the point, a lot of work regarding three analysis models i.e. cost, energy and sensing coverage analysis of heterogeneous WSN have been discussed in the literature. In paper [9], the cost model for evaluating the cost of heterogeneous network deployment is presented. Also, the authors reached a conclusion that heterogeneous deployment achieved higher coverage rate and lower deployment cost, keeping the number of nodes same. A cost based comparative study between different communication models is done by [10], where, authors described an optimal heterogeneous sensor deployment regarding battery energy and lower cost. Heinzelman et al. [11] provided an analysis of an optimal number of clusters of energy efficient clustering protocol and provided energy consumption model for different consumption schemes. Further, Duarte-Melo and Liu [12] presented the energy consumption model and quantified the optimal number of clusters based on the suggested model. Not only the cost and energy factors are significant, but also sensing coverage remains the primary focus of heterogeneous domain. Huang et al. discuss the worst case and best case coverage problems [13]. Different sensing coverage degree has been reviewed and simulated for different network locations [14]. A coverage configuration protocol proposed by Wang et al. [15] suggested a model for application based coverage. Howard et al. [16] proposed an initial deployment to maximize the coverage. None of the work in the literature has considered a tradeoff between three models. Lee et al. [17] proposed an optimal mixture of low-end and high-end nodes that can simultaneously optimize network lifetime and sensing coverage. The work can be applied to find a solution to the optimality problem between network lifetime and sensing operations for various levels of the heterogeneous network. None of the work in the literature combined the cost, energy and sensing coverage analysis models and provided the optimal number of high-end nodes for various levels of heterogeneity. The novelty of our work lies in finding the mathematical model for multi-objective deployment of heterogeneity.

\section{WORKING DESCRIPTION OF PROPOSED APPROACH}

Reactive algorithms are best suitable for event detection applications. The sensing nodes remain at sleep node till some event occurs, or the base station fires a query to a sensing node. Hence, there is no need to create the paths from the node to the base station in advance as these nodes will be using the paths only when the event is happening. Afterward, nodes will go to sleep mode again. This approach helps in saving the overhead to maintain the routing tables by the sensor nodes and eventually leading to energy savings. EE-SIR proposes the same approach of creating paths on a need basis. EE-SIR follows a similar approach as Source Initiated Reactive (SIR) [18] with better implementation regarding the primary routing strategy used, i.e. energy aware gossiping. EE-SIR allows source nodes to gossip their data (e.g., on event detection) as long as a route is not available. Sensing node selects the parent node by a parameter based on four factors viz. Distance to the base station, residual energy left; no of times that node has already been a parent node earlier, and the total neighboring nodes. These features make EE-SIR more reactive in the presence of dynamic network behavior, but more moderate in scenarios with demanding traffic. The originality of the stated approach is to provide the simulation and performance evaluation of EE-SIR algorithm on different levels of heterogeneous networks. Levels represent the types of nodes with different capabilities a system deploys e.g. 2-level means there are two kinds of nodes; one equipped with lower initial 
energy and another with higher initial energy. Similarly, 3level means that nodes with three types of energy levels are considered. Again, n-level means that n- levels of energy are examined. Adding heterogeneity would enhance the uniform dissipation of energy. If a node with higher capability is there in the vicinity of the sensing node, then that node will be selected as a parent node otherwise, one of the same capability nodes will be chosen by other three factors defined in the stated parameter. The working of the stated approach is as under:

\section{A. Initialization Phase}

In the first phase, all the nodes are divided randomly. In the initialization phase, the network is simulated for four deployments. First, the homogeneous deployment of the network, where all the similar nodes are deployed randomly in the network. 2-level heterogeneous network, where the network considers two types of nodes viz. Lower capability nodes and higher capability nodes. Similarly for 3-level and further for n-level.

\section{B. Neighboring Node Selection Phase}

This step selects the neighboring node of the sensing node. The sensing node selects the adjacent node by several factors like residual energy, neighboring nodes, distance to the base station, stability values. The number of times the node has been a leader node determines its stability value. To ensure the uniform energy drainage amongst the nodes, the role of the leader rotates among all the powerful nodes of the subnetwork. The neighboring leader node is selected using a Selection parameter $\mathrm{S}(1)$ :

$$
S(l)=\min _{s, i \in n} d(s, i)+\frac{E_{\text {residual }}}{E_{\text {total }}}+\frac{K}{1-K(r(\bmod (1 / K)))}+T(n)
$$

In (1), $\mathrm{s}$ is the sink node, $\mathrm{T}(\mathrm{n})$ is the number of neighboring nodes, $\mathrm{K}$ is the number of rounds that have already been done, and $\mathrm{r}$ is the current round. The third factor in the above equation ensures that if a leader node has been elected in the last $1 / \mathrm{k}$ round, it will not be selected in this round. After the selection of a neighboring node, the node maintains a routing table, which contains the id of the neighboring node selected. The process continues till the route to the base station is not formed.

The working of EE-SIR approach is as follows:

1) The sensing node that detects an event sends a control packet to all the neighboring nodes.

2) A control packet describing all the four entries i.e. distance to the base station, residual energy, no. of times the node has been selected earlier, no. of neighboring nodes is sent back to the sensing node.

3) Depending on the value of the parameter calculated from the four factors, sensing node selects the parent node to forward and route the sensed data.

4) The selected node is sent the sensed data, sensing node id and a timestamp.

5) The selected parent node in turn will send the control packet to all the neighboring nodes to select its parent, and the process is repeated till the base station is reached

\section{SYSTEM MODEL}

Before proceeding to discuss the performance characteristics of the stated approach, a discussion is laid on the underlying system models (viz. Energy Consumption, Cost model and Sensing coverage Mode) which have been used to analyze the same. Correspondingly, the analysis has been presented for 2-level, 3-level, and n-level heterogeneous network. In the case of a 2-level network, two different types of nodes are considered viz. Normal nodes and advanced nodes (having capabilities more than normal nodes). If there is a single advanced node in the network, then it is evident that it will be acting as a base station, and hence, this type of network can be treated as a homogenous network. On the other hand, if the number of advanced nodes is greater that one, then the particular network behaves like a 2-level heterogeneous network. Similarly, for a 3-level network, three types of nodes i.e. normal, advanced and super nodes are considered with their capabilities descending in the same order. If there is only a single sink, and the super node is acting as the sink, then this type of network can be analyzed as the 2-level heterogeneous network. Further, in an n-level network, the generalization for multiple levels of nodes representing various initial energy levels is done.

\section{A. Cost Analysis Model}

For the 2-type heterogeneous network, two types of nodes are assumed: Advanced nodes $(A)$ and normal nodes $(N)$. The total number of nodes to be deployed in the network depends on the cost constraint as Cost constraint $\geq n_{a} C_{a}+n_{n} C_{n}$ where $n_{a} C_{a}$ and $n_{n}, C_{n}$ are the number and cost of advanced nodes and normal nodes respectively.

$$
\mathrm{C}_{\text {total }}=n_{a} * C_{a}+n_{n} * C_{n}
$$

If the number of advanced nodes is one i.e. just the base station and $N_{n}{ }^{*}$ is the maximum number of normal sensor nodes that can be deployed in the network with a single advanced node i.e. base station, which can be determined by the following equation:

$$
C_{\text {total }}=C_{a}+N_{n}^{*} C_{n}
$$

Total number of normal nodes that can be deployed in a 2level heterogeneous network is:

$$
n_{n}=N_{n}{ }^{*}+\frac{C_{a}}{C_{n}}\left(1-n_{a}\right)
$$

For the 3-type heterogeneous network, three levels of nodes are deployed: Super $\operatorname{Nodes}(S)$, advanced nodes $(A)$ and normal nodes $(N)$. The total number of nodes to be deployed in the network depends on the cost constraint as Cost constraint $\geq n_{s} C_{s}+n_{a} C_{a}+n_{n} C_{n}$ where $n_{s}, n_{a}$ and $n_{n}$ are the numbers of super nodes, advanced nodes, and normal nodes respectively, and $C_{s}, C_{a}$ and $C_{n}$ are the costs of the respective sensor node. Total cost is determined from the following equation:

$$
\mathrm{C}_{\text {total }}=n_{s} * C_{s}+n_{a} * C_{a}+n_{n} * C_{n}
$$

If the number of super nodes is one i.e. just the base station, then the total cost is determined by: 


$$
C_{\text {total }}=C_{s}+N_{a}^{*} C_{a}+N_{n}^{*} C_{n}
$$

Where $\mathrm{N}_{\mathrm{a}}{ }^{*}, \mathrm{~N}_{\mathrm{n}}{ }^{*}$ is the maximum number of advanced and normal sensor nodes that can be deployed in the network with a single super node i.e. base station, which can be determined from the following equation:

$$
\begin{aligned}
& N_{n}{ }^{*}=n_{n}+\left(n_{a}-1\right) \frac{C_{a}}{C_{n}} \\
& N_{a}{ }^{*}=1+\left(n_{s}-1\right) \frac{C_{s}}{C_{a}}
\end{aligned}
$$

The number of advanced nodes in a 3-level heterogeneous network is determined by:

$$
n_{a}=\left(1-n_{s}\right) \frac{C_{s}}{C_{a}}+N_{a}{ }^{*}+\left(N_{n}{ }^{*}-n_{n}\right) \frac{C_{n}}{C_{a}}
$$

The number of normal nodes in a 3-level heterogeneous network is:

$$
n_{n}=N_{n}^{*}+\left(1-n_{a}\right) \frac{C_{a}}{C_{n}}
$$

For n-type heterogeneous network, where $\mathrm{n}$ different energy levels are considered:

$$
C_{\text {total }}=\sum_{i=1}^{n} n_{i} * C_{i}
$$

The cost is determined from numerous factors like energy, communication range and sensing range and other parameters which include memory and processing capacity. Under the prescribed cost constraint, if the node at higher end increases, then undeniably the number of nodes at lower end decreases that might have a direct impact on sensing coverage and network lifetime.

\section{B. Energy Analysis Model}

In this manuscript, the event detection model is assumed where a temporal correlation of events is considered. Furthermore, temporal correlation of real signals is one of the many statistical features which is efficaciously practiced for event detection in WSN applications. In addition to that, the communication model assumed is single-hop communication model in which every node is directly communicating with the base station. Energy consumption is cumulative of data processing, sensing, communication, amplifier energy that is related to the distance from the sink, and the path-loss exponent is assumed to be 2.The energy model used here in EE-SIR is based upon the goal of minimization of energy, whenever, there is some discrete event field. The model assumes that the main consumption of energy comes from the exchange of data messages, and a fixed amount of energy is used for control messages exchanged. All energy consumption values are collected from source to destination, and all calculations are based on an event field and not based on rounds of simulations as done by other researchers. by:

For 2-level heterogeneous network, initial energy is given

$$
E_{\text {total }}=n_{n} * E_{0}+n_{a} E_{0} *(1+\alpha)
$$

Effectively, this heterogeneous network has ' $\alpha m$ ' nodes amounting to ' $\alpha m$ ' times the additional energy in the network.

For 3-Level heterogeneous network, the total energy of the netw network is:
$E_{\text {total }}=n_{n} * E_{0}+n_{a} * E_{0} *(1+\alpha)+n_{s} * E_{o}(1+\beta)$

In multilevel heterogeneous WSN, the random energy 1 of sensor nodes is determined from a close-set $\left[E, E^{*}\left(1-\alpha_{\max }\right)\right]$, where ' $E$ ' is the lower bound and ' $\alpha_{\max }$ ' ascertain the value of the maximum energy. Initially, the node ' $s_{i}$ ' is equipped with an initial energy of ' $E_{0} *\left(1+\alpha_{i}\right)$ ', which is ' $\alpha_{i}$ ' times more energy than ' $E_{0}$ '. Hence, the total network energy is given by:

$$
\begin{aligned}
E_{\text {total }} & =\sum_{i=1}^{n} E_{0} *\left(1+\alpha_{i}\right) \\
\mathrm{E}_{\text {total }} & =\mathrm{E}_{0} * \sum_{\mathrm{i}=1}^{\mathrm{n}}\left(1+\alpha_{\mathrm{i}}\right)
\end{aligned}
$$

\section{Sensing Range Model}

For our simulation purpose, the sensing model assumed is the deterministic sensing model [19]. In this model, every sensor is involved in event detection. The event is detected by a node based on the condition that if the received signal is greater than the detection threshold. The event detection is based on the received strength independent of environmental factors and the node specifications. Sensing coverage is calculated as the aggregate information gathered from all the alive nodes in the network. Hence, sensing coverage of the network is defined as the sum of sensing coverage of all the nodes.

$$
S_{\text {sum }}=\sum_{i=1}^{n} \pi R_{i}^{2}
$$

Sensing coverage can be defined as the total information that can be extracted from all the sensor nodes deployed in the network. Sensing coverage covers both sensing coverage area and sensing coverage degree. Sensing coverage area infers the area of the network that is monitored by at least one functioning sensor. Coverage degree represents the overlapping coverage among the neighboring sensor nodes i.e. the average number of sensors that cover any network area.

$$
S_{\text {sum }}=S_{d} S_{a}
$$

For 2-level heterogeneous network, under prescribed cost constraint, total sensing coverage can be expressed as:

$$
\begin{gathered}
S_{\text {sum }}=n_{a} \pi R_{a}{ }^{2}+n_{n} \pi R_{n}{ }^{2} \\
=\pi\left(n_{a} R_{a}{ }^{2}+\left(N_{n}{ }^{*}-\frac{C_{a}}{C_{n}}\left(n_{a}-1\right) R_{n}{ }^{2}\right.\right. \\
=\pi\left(-\left(R_{n}{ }^{2} \frac{C_{a}}{C_{n}}-R_{a}{ }^{2}\right) n_{a}+\left(\frac{C_{a}}{C_{n}}+N_{n}{ }^{*}\right) R_{n}{ }^{2}\right) \\
=\pi\left(\gamma_{a} n_{a}+\gamma_{b}\right)
\end{gathered}
$$

Where $R_{n}$ and $R_{a}$ are the sensing range of advanced nodes and normal nodes.

For the 3-level heterogeneous network, the case is presented for three types of nodes viz. super nodes, advanced nodes and normal nodes having ranges $R_{s}, R_{a}$, and $R_{n}$ respectively.

$$
\begin{aligned}
S_{\text {sum }}=n_{s} \pi R_{s}{ }^{2}+n_{a} \pi R_{a}{ }^{2}+n_{n} \pi R_{n}{ }^{2} \\
=\pi\left[n_{s} R_{s}{ }^{2}+\left[\left(1-n_{s}\right) \frac{c_{s}}{c_{a}}+N_{a}{ }^{*}+\left(N_{n}{ }^{*}-\right.\right.\right. \\
\left.\left.\left.n_{n}\right) \frac{c_{n}}{c_{a}}\right] R_{a}{ }^{2}+n_{n} R_{n}{ }^{2}\right] \\
=\pi\left[n_{s} R_{s}{ }^{2}+R_{a}{ }^{2} \frac{c_{s}}{c_{a}}-n_{s} \frac{c_{s}}{c_{a}} R_{a}{ }^{2}+N_{a}{ }^{*} R_{a}{ }^{2}+\right. \\
\left.\quad\left(N_{n}{ }^{*}-n_{n}\right) \frac{c_{n}}{c_{a}} R_{a}{ }^{2}+\right] \\
=\pi\left(\gamma_{a} n_{s}+\gamma_{b}+\gamma_{c}\right)
\end{aligned}
$$


Where $\gamma_{a}, \gamma_{b}, \gamma_{c}$ are the factors determined from the above equation in order to simplify the equation.

Similarly for n-level heterogeneous network, the equation can be generalized as follows:

$$
\begin{aligned}
& S_{\text {sum }}=\sum_{i=1}^{n} \pi n_{i} R_{i}{ }^{2} \\
& S_{\text {sum }}=\pi\left(\gamma_{n} n_{n}+\sum_{i=1}^{n-1} \gamma_{i}\right)
\end{aligned}
$$

\section{NUMERICAL MODEL FOR OPTIMAL NUMBER OF High-} END NODES

\section{A. Impact of heterogeneity on network lifetime:}

All the three types of nodes are using the deterministic sensing model proposed by Ming et al. [22] but the sensing range of super nodes are higher than the advanced nodes. In this model, an event is detected if the received signal strength is greater than the sensing threshold set for event detection. For communication model, first order radio model as proposed by Wendi et al. [23] is used. The energy consumption of a single hop network can be calculated from two factors: one is independent of node range from sink and second factor that is entirely dependent on: can be calculated from Data Processing energy that is independent of the distance from the sink and the amplifier energy that is dependent on the distance from sink.

2-level Network

$$
E_{\text {total }}=E_{1}+E_{2} d^{2}
$$

$$
T=\frac{\pi E_{n} n_{a}}{\pi E_{1} n_{a}+S_{a} E_{2}}
$$

3-level Network

$$
T=\pi\left(\frac{E_{a} n_{s}}{\left(\pi E_{1} n_{s}+S_{a} E_{2}\right)}+\frac{E_{n} n_{a}}{\left(\pi E_{1} n_{a}+S_{a} E_{2}\right)}\right)
$$

n-level Network

$$
T=\pi \sum_{i=1}^{n} \frac{E_{i} n_{i-1}}{\left(\pi E_{1} n_{i-1}+S_{a} E_{2}\right)}
$$

\section{B. Impact of Heterogeneity on Sensing Coverage:}

Sensing coverage can be evaluated as the sensing coverage of all the alive nodes in the network. Hence, the total sensing coverage information can be calculated as:

$$
S=\sum_{t=0}^{T} S_{\text {sum }}\left(n_{a}, t\right)
$$

Where $T$ is the network lifetime and $S_{\text {sum }}$ is the total sensing coverage information at any point $t$, which indicates the sensing and the data gathering from all other sensors. The above equation is based on the assumption that each sensor is collecting the information from its sensing coverage and forwards the data to the base station.

Total sensing coverage can be expressed as:

$$
S=\pi\left(\gamma_{a} n_{a}+\gamma_{b}\right) \frac{\pi E_{n} n_{a}}{\pi E_{1} n_{a}+S_{a} E_{2}}
$$

To find out the optimal number of heterogeneous nodes that will maximize the sensing coverage, the equation will be differentiated on zero, Hence:

$$
n_{a-o p t}=\frac{1}{\sqrt{\gamma_{a}}} \sqrt{\left(\frac{S_{a} E_{2}}{\pi E_{1}}\right)^{2}+\gamma_{b} \frac{S_{a} E_{2}}{\pi E_{1}}}-\frac{S_{a} E_{2}}{\pi E_{1}}
$$

\section{3-Level network}

Sensing range can be defined as the total of all the sensing coverage of super nodes till the first node depletes its energy:

$$
S=\sum_{t=0}^{T} S_{\text {sum }}\left(n_{s}, t\right)
$$

Similarly as above, the optimal number of heterogeneous nodes for 3-level network can be evaluated by setting the derivative on $n_{s}$ as zero:

$$
\begin{aligned}
& \quad n_{s-o p t}=\pi\left(\gamma_{a} n_{s}+\gamma_{b}+\gamma_{c}\right) \pi\left(\frac{E_{a} n_{s}}{\left(\pi E_{1} n_{s}+S_{a} E_{2}\right)}+\right. \\
& \left.\frac{E_{n} n_{a}}{\left(\pi E_{1} n_{a}+S_{a} E_{2}\right)}\right)
\end{aligned}
$$

\section{$N$-level network}

The sensing range here will be the total of the highest level of energy node. If that is represented as $n_{i}$, then the sensing range of the network is:

$$
S=\sum_{t=0}^{T} S_{\text {sum }}\left(n_{i}, t\right)
$$

Similarly as above, the optimal number of highest level of nodes is expressed as:

$$
n_{i-o p t}=\pi \sum_{i=1}^{n} \frac{E_{i} n_{i-1}}{\left(\pi E_{1} n_{i-1}+S_{a} E_{2}\right)} \pi\left(\gamma_{n} n_{n}+\sum_{i=1}^{n-1} \gamma_{i}\right)
$$

\section{METHODS AND DISCUSSION}

The sensors are built using the design software Proteus created by Lab Center Electronics, which provides simulation for electronic circuits and control the schematic capture as well as PCB design. Few necessary presumptions of our routing protocol as given below:

a) All the available sensor nodes within the network have connectivity to communicate with each other or the base station (BS).

b) Random deployment of sensor node

c) All sensors dissipate their energy resource at the same rate at the time of iteration.

d) The primary factor of the total network lifetime is defined as the time span from the deployment to the instant when the first sensor node expires or when the entire sensor nodes perish.

e) The energy dissipation of sensing data and the energy dissipation for clustering are having negligible values and hence neglected.

f) One iteration round is defined as the time span when the Base Station collects information from all the sensor nodes and cluster head communicating the aggregation of data once. Each sensor node delivers only one sensed data with the same packet size that is defined for the network.

g) The sensor nodes within the network receive the data and combine one or more packets to produce a same-size resultant packet, and the number of data that needs to be sent by radio is reduced, because it is having many correlations between the data sensed by the different sensor nodes.

h) The sensor node energy dissipation of fusing one-bit data is a constant value.

Based on the above-identified problem and scope of work, following scenarios needs to be explored for arriving at a conclusive agreement or disagreement for identifying areas of improvement over the design of the new iterative algorithm. 
Homogeneous and heterogeneous are to be simulated. Heterogeneous are defined at three levels: 2-level, 3-level, and n-level.

TABLE I. SIMULATION PARAMETERS

\begin{tabular}{|l|l|}
\hline Parameters & Values \\
\hline $\mathrm{N} * \mathrm{~L}$ & 500 \\
\hline $\mathrm{E} 2 / \mathrm{E} 1$ & 0.2 \\
\hline $\mathrm{R}_{\mathrm{L}}$ & 0.1 \\
\hline $\mathrm{R}_{\mathrm{H}}$ & 0.2 \\
\hline $\mathrm{Sa}$ & $3 \pi$ \\
\hline $\mathrm{C}_{\mathrm{H}} / \mathrm{C}_{\mathrm{L}}$ & 10 \\
\hline
\end{tabular}

Impact of cost and Range ratio on Heterogeneity



2-level Network

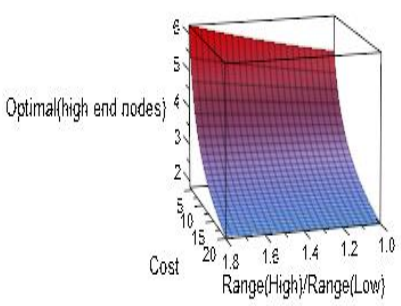

3-level Network
Fig. 1. Impact of Cost and Sensing Range on Optimal number of High nodes

The predictor variables i.e. cost(high nodes)/cost(low nodes) and range(high nodes)/range(low nodes) are displayed on $\mathrm{x}$ and $\mathrm{y}$ scales and $\operatorname{response}(\mathrm{z})$ variable i.e. the optimal number of high end nodes is represented using surface graph. Fig. 1 provides a clearer picture of the optimal number of high end nodes based on the cost ratio and range ratio of 2-level network. As seen in the graph as cost approaches to range ratio, number of optimal high end nodes increases steeply which in a way means that as cost decreases and range increases, it is viable to put more number of high end nodes in order to enhance the network lifetime. On the contrary, if the range ratio is not closer to cost ratio, it will not have a much impact on the heterogeneous deployment. Hence, it can be safely inferred that cost and sensing ratio affect the heterogeneous deployment only when the ratios are closer to each other.

Impact of Low cost nodes and Energy ratio on Heterogeneity

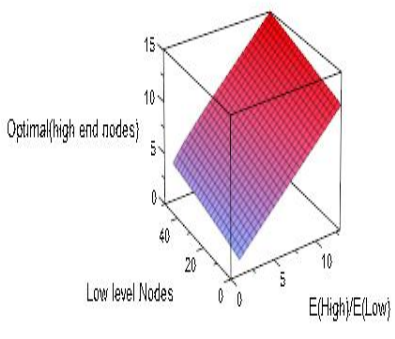

2-level Network

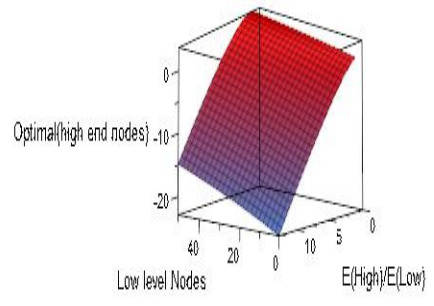

3-level Network
Fig. 2. Impact of Low Cost Nodes and Energy Ratio on Optimal Number

Fig. 2 shows the impact of number of low level nodes and energy ratio of advanced and super nodes with respect to the optimal number of advanced nodes. In 2-level heterogeneous network, the optimal number of advanced nodes varies almost linearly with the parameters mentioned. However, in 3-level heterogeneous network, the impact of the parameters is more than the 2-level network. Thus, more number of advanced nodes need to be deployed in a 2-level network as compared to the 3-level network.

Impact of Heterogeneity on Total Energy Spent: Total energy spent is considered to be the sum of energy consumed in sending and energy spent in receiving the packets. An increment of power consumption with the higher number of sources is depicted in the graph. Fig. 3 depicts that maximum energy consumption for homogenous is 61 Joules, which is almost double to 39.9 Joules consumed by the n-level heterogeneous network. 2-level and 3-level stay almost in the range of 50-55 Joules.

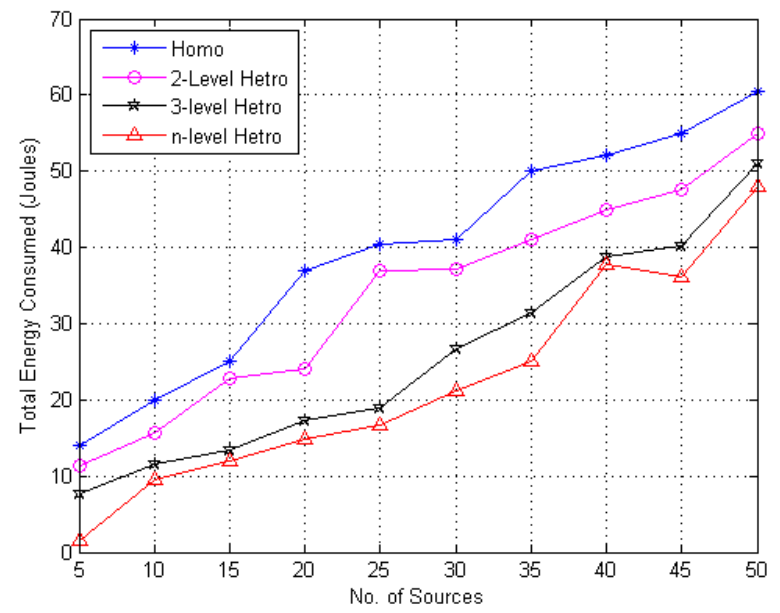

Fig. 3. Total Energy Spent

\section{CONCLUSION AND FUTURE SCOPE}

In this manuscript, cost analysis, sensing range, and energy analysis model for different heterogeneity levels in WSNs is proposed. Along with, a numerical model is presented that can provide an optimal number of high-end nodes to optimize network lifetime and sensing coverage simultaneously. This is an original work in the area of understanding the effect of energy heterogeneity and different heterogeneity levels in WSN. To the best of our knowledge, cost analysis for various levels has never been done in the literature. Extending the work to different levels of heterogeneity is the originality of our approach. The proposed approach is a novel approach to find out a mathematical model to calculate optimality for different levels of heterogeneous WSN. Discussed approach is well suited for resource-constrained networks operating with dynamic event detection. As future work, authors intend to explore more of heterogeneity parameters like computation, node capacities. The analysis models need to be applied for proactive strategies to verify the correctness of the other routing approach as well.

\section{REFERENCES}

[1] Gupta, P., \& Kumar, P. R. (2000). The capacity of wireless networks. Information Theory, IEEE Transactions on, 46(2), 388-404.

[2] Intel heterogeneous sensor networks research group. 
[3] Du, Xiaojiang, and Fengjing Lin. "Improving routing in sensor networks with heterogeneous sensor nodes." In Vehicular Technology Conference, 2005. VTC 2005-Spring. 2005 IEEE 61st, vol. 4, pp. 25282532. IEEE, 2005.

[4] Xu, Kaixin, Xiaoyan Hong, and Mario Gerla. "An ad hoc network with mobile backbones." In Communications, 2002. ICC 2002. IEEE International Conference on, vol. 5, pp. 3138-3143. IEEE, 2002.

[5] Mhatre, Vivek, and Catherine Rosenberg. "Homogeneous vs heterogeneous clustered sensor networks: a comparative study." In Communications, 2004 IEEE International Conference on, vol. 6, pp. 3646-3651. IEEE, 2004.

[6] Yu, Liyang, Neng Wang, Wei Zhang, and Chunlei Zheng. "Deploying a heterogeneous wireless sensor network." In Wireless Communications, Networking and Mobile Computing, 2007. WiCom 2007. International Conference on, pp. 2588-2591. IEEE, 2007.

[7] Mhatre, Vivek, and Catherine Rosenberg. "Design guidelines for wireless sensor networks: communication, clustering and aggregation." Ad hoc networks2, no. 1 (2004): 45-63.

[8] Kour, Harneet, and Ajay K. Sharma. "Hybrid energy efficient distributed protocol for heterogeneous wireless sensor network." International Journal of Computer Applications 4, no. 6 (2010): 1-5.

[9] Chakrabarty, Krishnendu, S. Sitharama Iyengar, Hairong Qi, and Eungchun Cho. "Grid coverage for surveillance and target location in distributed sensor networks." Computers, IEEE Transactions on 51, no. 12 (2002): 1448-1453.

[10] Lee, Jae-Joon, Bhaskar Krishnamachari, and CC Jay Kuo. "Impact of heterogeneous deployment on lifetime sensing coverage in sensor networks." In Sensor and Ad Hoc Communications and Networks, 2004. IEEE SECON 2004. 2004 First Annual IEEE Communications Society Conference on, pp. 367-376. IEEE, 2004.

[11] Wendi Rabiner Heinzelman, A. Chandrakasan and H. Balakrishnan. 2000. Energy-Efficient Communication Protocol for Wireless Microsensor Networks. Proceedings of the 33rd Hawaii International Conference on System Sciences (HICSS '00): 1-10.
[12] Duarte-Melo, Enrique J., and Mingyan Liu. "Analysis of energy consumption and lifetime of heterogeneous wireless sensor networks." In Global Telecommunications Conference, 2002. GLOBECOM'02. IEEE, vol. 1, pp. 21-25. IEEE, 2002.

[13] Huang, Chi-Fu, and Yu-Chee Tseng. "The coverage problem in a wireless sensor network." Mobile Networks and Applications 10, no. 4 (2005): 519-528.

[14] Du, Xiaojiang, and Fengjing Lin. "Maintaining differentiated coverage in heterogeneous sensor networks." EURASIP Journal on Wireless Communications and Networking 2005, no. 4 (2005): 565-572.

[15] Wang, Xiaorui, Guoliang Xing, Yuanfang Zhang, Chenyang Lu, Robert Pless, and Christopher Gill. "Integrated coverage and connectivity configuration in wireless sensor networks." In Proceedings of the 1st international conference on Embedded networked sensor systems, pp. 28-39. ACM, 2003.

[16] Howard, Andrew, Maja J. Matarić, and Gaurav S. Sukhatme. "Mobile sensor network deployment using potential fields: A distributed, scalable solution to the area coverage problem." In Distributed Autonomous Robotic Systems 5, pp. 299-308. Springer Japan, 2002.

[17] Lee, Jae-Joon, Bhaskar Krishnamachari, and CC Jay Kuo. "Impact of heterogeneous deployment on lifetime sensing coverage in sensor networks." In Sensor and Ad Hoc Communications and Networks, 2004. IEEE SECON 2004. 2004 First Annual IEEE Communications Society Conference on, pp. 367-376. IEEE, 2004.

[18] Sharma, Nonita, and Ajay K. Sharma. "Comparative Analysis of SIR and SID in Wireless Sensor Networks Using Temporal Correlation." network 1, no. 2: 3-4.

[19] Jellali, Z. ; Atallah, L.N. ; Cherif, S., "A study of deterministic sensors placement for sparse events detection in WSN based on Compressed Sensing," Proceedings of International conference on Communication and Networking, COMNET 2014,pp. 1-5, March 2014. 